\title{
Saúde mental dos profissionais da saúde na pandemia do coronavírus (Covid-19)
}

Mental health of health care professionals in the coronavirus pandemic (Covid-19)

\section{Salud mental de los profesionales de la salud en la pandemia de coronavirus}

(Covid-19)

Carolina Meira Moser $\mathbb{D}^{\mathrm{a}}$, Gabriela Carneiro Monteiro ${ }^{\mathrm{a}}$, Joana Correa de Magalhães Narvaez $\mathbb{D}^{\mathrm{b}}$, Felipe Ornell $\mathbb{D}^{c}$, Vitor Crestani Calegaro $\mathbb{( D}^{\mathrm{d}}$, Ana Margareth Siqueira Bassols $\mathbb{D}^{\mathrm{a}}$, Pricilla Braga Laskoski $\mathbb{D}^{\mathrm{a}}$, Simone Hauck (1D)

a Universidade Federal do Rio Grande do Sul, Programa de Pós-Graduação em Psiquiatria e Ciências do Comportamento - Laboratório de Psiquiatria Psicodinâmica - Porto Alegre/RS - Brasil. ' ${ }^{b}$ Unversidade Federal de Ciências da Saúde de Porto Alegre, Curso de Psicologia - Porto Alegre/RS - Brasil. ' Universidade Federal do Rio Grande do Sul, Programa de Pós-Graduação em Psiquiatria e Ciências do Comportamento - Hospital de Clínicas de Porto Alegre, Centro de Pesquisa em Álcool e Drogas - Porto Alegre/RS - Brasil. ${ }^{d}$ Universidade Federal de Santa Maria, Departamento de Neuropsiquiatria - Santa Maria/RS - Brasil.

Instituição: Universidade Federal do Rio Grande do Sul, Programa de Pós-Graduação em Psiquiatria e Ciências do Comportamento - Laboratório de Psiquiatria Psicodinâmica - Porto Alegre/RS - Brasil

DOI 10.5935/2318-0404.20210009

O presente trabalho foi realizado com apoio da Coordenação de Aperfeiçoamento de Pessoal de Nível Superior - Brasil (CAPES) - Código de Financiamento 001.

\section{Resumo}

Introdução: A pandemia do coronavírus (Covid-19) impôs desafios adicionais aos profissionais da saúde (PS), potencializando o risco de sofrimento psíquico. Objetivo: Avaliar o perfil sociodemográfico e a saúde mental de uma amostra de PS do Brasil durante a pandemia do Covid-19. Métodos: Estudo transversal online realizado durante 1 mês, entre maio e junho de 2020. Sintomas depressivos foram avaliados pelo Patient Health Questionnaire 9 (PHQ-9) e o nível de burnout pelo Copenhagen Burnout Inventory (CBI). Resultados: 1054 PS foram incluídos, sendo 34,5\% médicos, 19,1\% técnicos de enfermagem, 14,2\% enfermeiros e 11,9\% psicólogos. Mais da metade da amostra total apresentou escores $\geq 50$ no domínio de burnout pessoal (PB) da CBI, indicativo de alto nível de burnout, e escores no PHQ-9 sugestivos de depressão clinicamente significativa, 
sendo estes índices mais elevados entre os técnicos de enfermagem (68,2\% com PB $\geq 50$ e 68,7\% com PHQ$9 \geq 9$ ) e os PS da linha de frente (61,3\% com PB $\geq 50$ e $58 \%$ com PHQ-9 $\geq 9)$. Os técnicos de enfermagem apresentaram escores PB $(58,4 \pm 20,9)$ e do domínio de burnout relacionado ao trabalho (WB $=51,0 \pm 21,1$ ) superiores aos dos médicos ( $P B=48,2 \pm 19,9$ e $W B=44,2 \pm 19,1)$ e dos psicólogos ( $P B=44,2 \pm 17,4$ e $W B=41,2$ $\pm 16,7) p<0,001$. Conclusão: Os elevados níveis de burnout e depressão, mais preocupantes entre os técnicos de enfermagem, corroboram a vulnerabilidade dos PS ao sofrimento emocional no contexto do atendimento à Covid-19, demonstrando a urgência de intervenções específicas.

Palavras-chaves: Infecções por coronavirus; Pessoal de saúde; Saúde mental; Depressão; Esgotamento profissional

\section{Abstract}

Introduction: The coronavirus pandemic (Covid-19) posed additional challenges to health care professionals (PS), increasing the risk of psychological distress. Objective: To evaluate the sociodemographic profile and mental health of a sample of PS in Brazil during the Covid-19 pandemic. Methods: Cross-sectional online study conducted during 1 month, between May and June 2020. Depressive symptoms were assessed by the Patient Health Questionnaire 9 (PHQ-9) and the level of burnout by the Copenhagen Burnout Inventory (CBI). Results: 1054 PS were included, being 34.5\% physicians, 19.1\% nursing technicians, $14.2 \%$ nurses and $11.9 \%$ psychologists. More than half of the total sample had scores $\geq 50$ in the the personal burnout (PB) domain of $\mathrm{CBI}$, indicative of a high level of burnout, and PHQ-9 scores suggestive of clinically significant depression, these rates being higher among nursing technicians (68.2\% with BP $\geq 50$ and $68.7 \%$ with PHQ-9 $\geq 9$ ) and frontline PS (61.3\% with BP $\geq 50$ and $58 \%$ with PHQ-9 $\geq 9$ ). Nursing technicians had PB scores $(58.4 \pm 20.9)$ and workrelated burnout domain scores $(W B=51.0 \pm 21.1$ ) higher than those of physicians ( $P B=48.2 \pm 19.9$ and $W B$ $=44.2 \pm 19.1)$ and psychologists $(P B=44.2 \pm 17.4$ and $W B=41.2 \pm 16.7) p<0.001$. Conclusion: The high levels of burnout and depression, most worrying among nursing technicians, corroborate the vulnerability of the PS to emotional suffering in the context of the Covid-19 pandemic. Specific interventions are urgent.

Keywords: Mental health; Depression; Burnout professional; Coronavirus infections; Allied health personnel

\section{Resumen}

Introducción: La pandemia de coronavirus (Covid-19) planteó desafíos adicionales a los profesionales de la salud (PS), aumentando el riesgo de angustia psicológica. Objetivo: Evaluar el perfil sociodemográfico y la salud mental de una muestra de PS en Brasil durante la pandemia Covid-19. Métodos: Estudio transversal en línea realizado durante 1 mes, entre mayo y junio de 2020. Los síntomas depresivos fueron evaluados por el Patient Health Questionnaire 9 (PHQ-9) y el nivel de burnout por el Copenhagen Burnout Inventory (CBI). Resultados: Se incluyeron 1054 PS, siendo 34,5\% médicos, 19,1\% técnicos de enfermería, 14,2\% enfermeras y $11,9 \%$ psicólogos. Más de la mitad de la muestra total tuvo puntajes $\geq 50$ en el dominio de Burnout personal (PB) de $\mathrm{CBI}$, indicativo de un alto nivel de Burnout, y puntajes PHQ-9 sugestivos de depresión clínicamente 
significativa, siendo estas tasas más altas entre los técnicos de enfermería $(68,2 \%$ con PB $\geq 50$ y $68,7 \%$ con PHQ-9 $\geq 9)$ y el PS de primera línea (61,3\% con PB $\geq 50$ y $58 \%$ con PHQ-9 $\geq 9)$. Los técnicos de enfermería tuvieron puntuaciones de PB $(58,4 \pm 20,9)$ y el dominio de burnout laboral $(W B=51,0 \pm 21,1)$ superior al de los médicos ( $P B=48,2 \pm 19,9$ y $W B=44,2 \pm 19,1)$ y psicólogos $(P B=44,2 \pm 17,4$ y $W B=41,2 \pm 16,7) p$ $<0,001$. Conclusión: Los altos niveles de burnout y depresión, que son más preocupantes entre los técnicos de enfermería, corroboran la vulnerabilidad del PS al sufrimiento emocional en el contexto de la atención al Covid-19, demostrando la urgencia de intervenciones específicas.

Palabras clave: Infecciones por coronavirus; Salud mental; Depresión; Agotamiento profesional; Salud; Técnicos medios en salud

\section{Introdução}

A pandemia do Covid-19, cientificamente chamado de SARS-CoV-2, trouxe grandes desafios aos sistemas de saúde mundiais, exigindo estratégias complexas e políticas públicas de prevenção, contenção, diagnóstico e tratamento. Nesse contexto, os profissionais da saúde (PS) constituem um grupo de alto risco de exposição ao SARS-CoV-2, sendo imperativo o apoio e monitoramento adequados do bem-estar desses indivíduos ${ }^{1-4}$, já que aos riscos biológicos somam-se os efeitos da pandemia na saúde mental ${ }^{5,6}$.

Diante do aumento exponencial da demanda de atendimentos, os PS enfrentam longas jornadas de trabalho, muitas vezes com recursos abaixo do ideal e infraestrutura precária ${ }^{7}$. O estresse decorrente da sobrecarga de trabalho e da falta de protocolos clínicos ou tratamentos bem estabelecidos para o manejo dos pacientes infectados torna as equipes de saúde particularmente vulneráveis ao aumento da ansiedade e sofrimento psíquico ${ }^{7-9}$. Este fenômeno pode potencializar o risco de desenvolvimento de transtornos psiquiátricos ${ }^{10,11}$ e agravar os sintomas em indivíduos com transtornos pré-existentes ${ }^{7,12-15}$. Além disso, o medo de contágio e da possibilidade de contaminar familiares, amigos ou colegas ${ }^{8,16}$ são estressores adicionais importantes, que podem levar ao isolamento da família, modificação da rotina e estreitamento da rede de apoio social ${ }^{3}$.

Estudos anteriores já demonstraram que epidemias passadas foram seguidas de impactos psicossociais drásticos e mais duradouros que o próprio surto ${ }^{17,18}$. Durante o surto de Síndrome Respiratória Aguda Severa (SARS) em 2003, 18 a 57\% dos PS experimentaram problemas emocionais e sintomas psiquiátricos graves durante e após o evento ${ }^{19}$. Em 2015, no decorrer da Síndrome Respiratória do Oriente Médio (MERS), os estados disfóricos e o estresse evidenciados nas equipes de saúde foram preditores de condutas equivocadas, atraso por falha na comunicação e absenteísmo, entre outros. A ocorrência da síndrome de burnout também foi relatada por PS envolvidos na assistência de indivíduos infectados na epidemia de outro tipo de coronavírus ocorrido na Coréia em $2016^{20}$. Além disso, a traumatização vicária, fenômeno no qual os PS experimentam sintomas semelhantes aos dos pacientes devido à exposição contínua, também é comum durante catástrofes. Os principais sintomas da traumatização indireta são a perda de apetite, fadiga, declínio físico, distúrbios do sono, irritabilidade, desatenção, distanciamento emocional ("incapacidade de sentir"), medo e desespero ${ }^{17}$. 
Estudos recentes sobre os efeitos do Covid-19 na saúde mental das equipes de saúde ${ }^{21-23}$, incluindo revisões sistemáticas ${ }^{24-28}$, demonstram que se trata de uma problemática emergente: índices preocupantes de depressão, ansiedade, insônia e estresse foram encontrados. Entre os fatores de risco já identificados para maior impacto psicológico do Covid-19 nos PS estão: ser do sexo feminino, enfermeiro, baixo nível socioeconômico, isolamento social e alto risco de contaminação ${ }^{12}$. Como fatores protetores, estão o apoio familiar, presença de recursos médicos suficientes, acesso à informação atualizada e confiável, assim como medidas de precaução, como higiene de mãos e uso de máscaras ${ }^{12,29}$.

Níveis elevados de burnout e trauma secundário foram reportados na Itália durante a pandemia ${ }^{30}$. Isso também ocorreu no Brasil, onde foram identificados elevados índices de burnout entre médicos ${ }^{31}$, assim como altas taxas de sintomas de depressão, ansiedade e estresse ${ }^{31}$ entre médicos e outras categorias de PS ${ }^{32}$. Além disso, trabalhadores de serviços essenciais brasileiros, não apenas profissionais da saúde, apresentaram índices de depressão e ansiedade superiores aos reportados na Espanha ${ }^{33}$. Considerando este cenário, é importante avaliar a realidade da população dos PS brasileiros no que se refere a taxas de burnout e depressão, bem como aos fatores associados à maior vulnerabilidade psicossocial. De fato, o melhor entendimento da saúde mental da equipe assistencial, bem como do seu impacto no cuidado dos pacientes, é urgente, havendo necessidade da implementação de ações imediatas. Dessa forma, o presente estudo teve como objetivo avaliar o perfil sociodemográfico e aspectos relacionados à saúde mental de uma amostra de profissionais da saúde do Brasil durante a pandemia do Covid-19.

\section{Método}

\section{Desenho e Participantes}

Trata-se de um estudo transversal online. O recrutamento dos participantes foi realizado por meio do método "snowball" (bola de neve) direcionado aos PS do Brasil durante um mês (22 de maio a 22 de junho de 2020). Não houve direcionamento específico a PS envolvidos na linha de frente da assistência de pacientes com Covid-19. Foram incluídas 17 categorias profissionais apontadas pelo Ministério da Saúde na Portaria número 639 de 31 de março de 2020 para cadastramento na ação estratégica voltada ao enfrentamento da pandemia do Covid-19 diretamente envolvidas no cuidado de pacientes humanos: Médico(a), Enfermeiro(a), Técnico(a) de enfermagem, Psicólogo(a), Fisioterapeuta, Fonoaudiólogo(a), Terapeuta Ocupacional, Nutricionista, Assistente Social, Educador(a) Físico, Odontólogo(a), Técnico(a) em radiologia, Farmacêutico(a), Biomédico(a), Biólogo(a), Biotecnólogo(a) e Agente Comunitário de Saúde.

\section{Coleta de dados e Aspectos Éticos}

A coleta dos dados foi iniciada após a aprovação do Comitê de Ética em Pesquisa do Hospital de Clínicas de Porto Alegre e Comissão Nacional de Ética em Pesquisa (CAAE número 30745020.5.0000.5327). Os autores assinaram um Termo de Compromisso para Uso de Dados, consentindo que as informações seriam utilizadas única e exclusivamente para fins acadêmicos e científicos previstos no estudo. 
Os participantes responderam a um questionário virtual formulado na plataforma "SurveyMonkey" que foi veiculado em redes sociais (Facebook e Instagram), além de compartilhado através de instituições e profissionais das diferentes áreas por conveniência. Os questionários foram divulgados nas páginas do Facebook e Instagram através do mecanismo de impulsionamento patrocinado direcionado a indivíduos de todas as idades que vivem no Brasil. A escolha por um questionário eletrônico, assim como a utilização da citada plataforma para divulgação dos questionários, foi baseada em experiências prévias positivas com essa metodologia ${ }^{34}$ bem como em função do atual contexto de pandemia e necessidade de medidas de isolamento/ distanciamento social.

Na introdução do questionário, foi apresentado o Termo de Consentimento Livre e Esclarecido, explicitando que o anonimato estaria garantido e que os dados seriam analisados apenas em conjunto. Os participantes foram informados sobre eventuais riscos envolvidos na participação dessa pesquisa. Além disso, no final do questionário disponibilizamos os contatos do Centro de Valorização à Vida (CVV), bem como telefone, e-mail e endereço da equipe de pesquisa para obter informações sobre onde buscar atendimento. Também foi oferecida a possibilidade de ter acesso aos resultados do estudo e a material psicoeducativo em saúde mental através do e-mail específico da pesquisa. Ao aceitar responder o questionário, o indivíduo estaria concordando em participar do estudo.

\section{Instrumentos}

Os dados foram coletados por meio de um questionário contemplando dados sociodemográficos, ideação suicida, assistência a indivíduos com suspeita ou diagnóstico de coronavírus e percepção de risco.

- Questionário sobre a Saúde do(a) Paciente (Patient Health Questionnaire 9-PHQ-9) 35 : instrumento de aplicação rápida, que rastreia indivíduos em maior risco para o episódio depressivo maior. Tanto possibilita o diagnóstico do transtorno, quanto a classificação do grau de intensidade dos sintomas. Esse questionário é usado para rastrear sintomas nas últimas 2 semanas, avaliando a presença de sintomas depressivos ${ }^{36}$, conforme protocolo do DSM-5, por meio de uma escala do tipo Likert composta por nove perguntas categorizadas em quatro opções de respostas, que vão de "não, nenhum dia" (zero pontos) a "quase todos os dias" (3 pontos); no total, os valores resultam de zero a 27 pontos. Assim, quanto maior a pontuação, maior a gravidade dos sintomas depressivos. Neste estudo, foi adotado o ponto de corte $\geq 9$, conforme recomendado ${ }^{37,38}$. O PHQ9 teve suas propriedades de rastreamento validadas no Brasil para população geral em $2013^{37}$. Esta escala tem demonstrado boas características psicométricas e operacionais, com sensibilidade entre 77 e $98 \%$ e especificidade de 75 a $80 \%$, tendo sido validada para população de adultos e idosos ${ }^{38}$.

- Inventário de Burnout de Copenhagen (The Copenhagen Burnout Inventory - CBI): instrumento desenvolvido por Kristensen e colegas que considera a fadiga e a exaustão como os principais constructos do burnout $^{39}$. O CBI consiste em 19 itens divididos em três domínios a partir dos quais a exaustão emocional pode surgir: pessoal ( 6 itens), relacionada ao trabalho ( 7 itens) e relacionada ao cliente/paciente (6 itens). Burnout pessoal (PB) é o grau de exaustão física e psicológica que uma pessoa pode vivenciar, não necessariamente 
relacionada à sua ocupação. Burnout relacionado ao trabalho (WB) é o grau em que o desgaste físico e psicológico é percebido em relação às atividades laborais. O burnout relacionado ao cliente/paciente (CB) é o nível de exaustão que é atribuído ao relacionamento profissional com o cliente/paciente. Trata-se de um questionário auto-aplicável em escala Likert de 5 pontos: "Sempre" ou "Em um grau muito alto" (100 pontos), "Frequentemente" ou "Em um grau alto" (75 pontos), "Às vezes" ou "Em algum grau" (50 pontos), "Raramente" ou "Em baixo grau" (25 pontos) e "Nunca" ou "Em um grau muito baixo" (0 pontos). Os escores de cada domínio da CBI são obtidos através da média de cada subescala. Quanto maiores os escores, maiores os níveis de burnout. Apesar da recomendação do autor de usar os escores de forma dimensional, valores iguais ou superiores a 50 no domínio pessoal são considerados sugestivos da presença de burnout clinicamente significativo. Entre as vantagens da $\mathrm{CBI}$ em relação ao Maslach Burnout Inventory (MBI) ${ }^{40}$ estão o acesso (instrumento de domínio público), a avaliação de um mesmo constructo global (fadiga e exaustão) em diferentes contextos e a possibilidade de ser utilizada em qualquer tipo de trabalho/ocupação, não ficando restrita a setores de serviços humanos. O CBI está adaptado e validado para o português brasileiro ${ }^{41}$.

Ideação Suicida:

As perguntas relacionadas a suicídio no presente estudo foram baseadas nos instrumentos Columbia Suicide Severity Rating Scale (C-SSRS) ${ }^{42}$ e Beck Scale for Suicide Ideation (BSI) ${ }^{43}$ abaixo descritos, que são frequentemente utilizados para avaliação do suicídio na literatura. Foram selecionados os questionamentos mais importantes com o objetivo de avaliar a prevalência de ideação suicida nesta população, visto que a inclusão do instrumento completo poderia tornar o questionário muito longo, prejudicando a adesão dos participantes à pesquisa.

- Columbia Suicide Severity Rating Scale (C-SSRS) ${ }^{42}$ : instrumento utilizado para identificar e avaliar indivíduos em risco de suicídio. As perguntas são formuladas para serem usadas em formato de entrevista, mas podem ser respondidas como uma medida de autorrelato, se necessário. O C-SSRS mede quatro construtos: a gravidade da ideação, a intensidade da ideação, comportamento e letalidade. O C-SSRS é composto por dez categorias, todas com perguntas de respostas binárias (sim / não) para indicar a presença ou ausência do comportamento. As categorias incluídas consistem em desejo de morrer, pensamentos suicidas ativos não específicos, ideação suicida ativa com qualquer método (não planejado) sem intenção de agir, ideação suicida ativa com alguma intenção de agir, sem plano específico; ideação suicida ativa com plano e intenção específicos; atos ou comportamento preparatório; tentativa abortada; tentativa interrompida; tentativa real (não fatal); e suicídio completo. Não há um ponto de corte estabelecido.

- Beck Scale for Suicide Ideation (BSI) ${ }^{44}$ : instrumento auto-aplicável de 21 itens para avaliar a intensidade de atitudes, comportamentos e planos suicidas na última semana. Apresenta cinco itens de triagem: três que avaliam o desejo de viver ou o desejo de morrer e dois que avaliam o desejo de tentativa de suicídio. Se o entrevistado relatar qualquer desejo ativo ou passivo de cometer suicídio, então 14 itens adicionais são administrados. Os primeiros 19 itens apresentam três opções de resposta classificadas de acordo com a 
intensidade da tendência suicida e pontuados de acordo com escala Likert de 3 pontos ( 0 a 2). A pontuação dos itens é somada para produzir um escore total, que varia de 0 a 38. Quanto maior o escore, maior o risco. No entanto, não são recomendados pontos de corte específicos. O instrumento apresenta validade preditiva para suicídio consumado.

\section{Análise estatística}

As análises estatísticas foram realizadas utilizando o software SPSS (versão 21). Análises descritivas foram realizadas com todas as variáveis demográficas e clínicas. As conclusões e generalizações se basearam na análise inferencial dos dados. As variáveis quantitativas foram descritas por média e desvio padrão e as categóricas por frequências absolutas e relativas. Para comparar médias, os testes t-student para amostras independentes ou Análise de Variância (ANOVA) complementada por Tukey foram aplicados. Para avaliar as associações entre as variáveis contínuas e ordinais, os testes da correlação de Pearson ou Spearman foram utilizados. A associação entre as variáveis categóricas foi avaliada pelo teste qui-quadrado $(\chi 2)$ de Pearson. Para as análises foi considerado $p<0,05$ como significativo.

\section{Resultados}

O estudo transversal teve início em 22 de maio de 2020, data em que o questionário foi divulgado através das mídias sociais e direcionado para profissionais da saúde de todo o Brasil. Obtivemos um total de 1256 participantes ao longo de 1 mês de coleta. Destes, 202 foram excluídos por não responderem às questões referentes à avaliação de sintomas de burnout. Em comparação aos participantes incluídos nas análises, os indivíduos excluídos foram mais jovens, com menos tempo de profissão e menor carga horária de trabalho semanal. Esse grupo também apresentou maior proporção de renda familiar abaixo de 1.500 reais, de indivíduos do grupo de alto risco para Covid-19 e das categorias profissionais de técnico de enfermagem, biomédico e biotecnólogo.

Dos 1054 participantes incluídos nas análises, tivemos uma maior proporção de médicos (34,5\%), técnicos de enfermagem $(19,1 \%)$, enfermeiros (14,2\%) e psicólogos (11,9\%) (Tabela 1). A maioria foi do sexo feminino (81\%), cor branca $(74,8 \%)$, casado ou em união estável $(57,2 \%)$, renda familiar acima de 10.000 reais $(39,6 \%)$ e residentes na região sul do Brasil (61,9\%). A média de idade foi de 41,7 anos, tempo de profissão de 15 anos e carga semanal de 37,6 horas de trabalho (Tabela 1 ).

Para a comparação das variáveis entre as categorias profissionais, foram agrupadas as categorias menos frequentes da amostra em um único grupo, designado "Outros" (Tabela 2). A categoria dos médicos apresentou maior proporção de indivíduos do sexo masculino em relação aos demais grupos. A proporção de profissionais da cor branca foi maior nas categorias dos médicos e psicólogos em comparação à dos técnicos de enfermagem. A proporção de casados ou em união estável foi maior entre os médicos e a proporção de separados ou divorciados foi maior entre os enfermeiros e técnicos de enfermagem. A maior renda familiar foi encontrada entre os médicos e a menor entre os técnicos de enfermagem. Uma maior proporção de profissionais 
da região sudeste foi encontrada entre os enfermeiros e técnicos de enfermagem. O tempo de profissão não foi diferente entre os médicos, enfermeiros, técnicos de enfermagem e psicólogos. A carga horária semanal de trabalho também não foi diferente entre médicos, enfermeiros e técnicos de enfermagem, sendo inferior entre os psicólogos e no grupo dos outros profissionais da saúde (Tabela 2).

Entre os técnicos de enfermagem, em comparação às demais categorias profissionais, uma proporção maior já havia tido diagnóstico confirmado de Covid-19 (10\%) e era do grupo de risco (36.3\%) (Tabela 3). Também foi a categoria que, juntamente com os enfermeiros e os médicos, esteve mais envolvida na assistência direta a pacientes com Covid-19 (59.2\%) e a que apresentou maior proporção de profissionais que referiram terem sido coagidos a realizar atividade para a qual não se sentiam capacitados $(20,1 \%)$. No entanto, em relação a essa última variável, a diferença encontrada não foi estatisticamente significativa (tabela 3).

Entre os médicos, houve uma maior proporção de indivíduos em tratamento de saúde mental (29.4\%) e com história de diagnóstico psiquiátrico (40.1\%). Entre enfermeiros e técnicos de enfermagem, uma maior proporção referiu não estar em tratamento atual para saúde mental, mas sentir que precisaria (28.7\% e 27.9\%, respectivamente). A categoria dos técnicos de enfermagem foi a que mais apresentou história de tentativa de suicídio (12.7\%) entre todas as categorias, e também a que mais apresentou trauma na infância (40.9\%), taxa similar à encontrada entre os psicólogos (37.4\%) (tabela 4).

No que se refere às taxas de burnout, um terço da amostra total referiu sentir-se em estado de burnout (Tabela 4) e mais da metade apresentou escores sugestivos de alto nível de burnout (valores $\geq 50$ na escala PB da Copenhagen) (Tabela 5). Conforme descrito na tabela 5, os técnicos de enfermagem apresentaram escores PB e WB superiores aos dos médicos e dos psicólogos, sem diferenças em relação aos enfermeiros e outras categorias profissionais. Os escores de burnout relacionado ao cliente/paciente (CB) foram semelhantes entre os grupos, exceto entre os psicólogos, que apresentaram os menores escores nessa dimensão (Tabela 5).

Com relação a sintomas depressivos, metade da amostra total apresentou escores de PHQ sugestivos de depressão clinicamente significativa (Tabela 5). Entre os técnicos de enfermagem, esta proporção foi de 68,7\%, semelhante à proporção encontrada em alto nível de burnout $(68,2 \%$ com PB $\geq 50)$ nesta categoria profissional.

Conforme descrito na tabela 6, os níveis de burnout e depressão de nossa amostra foram diferentes de acordo com o nível de exposição à assistência de indivíduos infectados com COVID- 19. Maior proporção de PS "da linha de frente" (assistência direta) considerou estar em síndrome de burnout (50,8\%) em comparação aos PS prestando assistência indireta (23,5\%) e aos PS que não estavam prestando assistência (25,7\%). Além disso, os escores de burnout nos domínios PB, WB e CB e os escores do PQH-9 dos PS envolvidos na assistência direta a pacientes com COVID foram superiores aos escores dos PS que não estavam prestando assistência. Em comparação aos PS envolvidos na assistência indireta, os profissionais da "linha de frente" apresentaram escores significativamente superiores nos domínios de burnout PB e WB (tabela 6). 
Tabela 1. Caracterização da amostra total.

\begin{tabular}{|c|c|}
\hline Variáveis & $\mathrm{n}=1054 *$ \\
\hline \multicolumn{2}{|l|}{ Sexo - n(\%) } \\
\hline Masculino & 200 (19) \\
\hline Feminino & $854(81)$ \\
\hline Idade - média \pm DP & $41.7(10.8)$ \\
\hline \multicolumn{2}{|l|}{ Cor - n(\%) } \\
\hline Branco & $788(74.8)$ \\
\hline Pardo & $182(17.3)$ \\
\hline Preto & $57(5.4)$ \\
\hline Amarelo & $16(1.5)$ \\
\hline Indígena & $4(0.4)$ \\
\hline Outro & $7(0.7)$ \\
\hline \multicolumn{2}{|l|}{ Estado civil - n(\%) } \\
\hline Casado(a)/ União Estável & $603(57.2)$ \\
\hline Separado(a) / Divorciado(a) & $129(12.2)$ \\
\hline Solteiro(a) com namorado(a) & $159(15.1)$ \\
\hline Solteiro(a) sem namorado(a) & 149 (14.1) \\
\hline Viúvo(a) & $14(1.3)$ \\
\hline \multicolumn{2}{|l|}{ Renda familiar - n(\%) } \\
\hline Até $1.500,00$ reais & $57(5.4)$ \\
\hline De 1.500 a 3.000 reais & $173(16.4)$ \\
\hline De 3.000 a 5.000 reais & $186(17.6)$ \\
\hline De 5.000 a 10.000 reais & $221(21)$ \\
\hline Acima de 10.000 reais & 417 (39.6) \\
\hline \multicolumn{2}{|l|}{ Região do país- n(\%) } \\
\hline Norte & $25(2.4)$ \\
\hline Nordeste & 109 (10.3) \\
\hline Centro oeste & $44(4.2)$ \\
\hline Sudeste & $224(21.3)$ \\
\hline Sul & $652(61.9)$ \\
\hline \multicolumn{2}{|l|}{ Categoria profissional- n(\%) } \\
\hline Médico & $364(34.5)$ \\
\hline Enfermeiro(a) & $150(14.2)$ \\
\hline Técnico de enfermagem & $201(19.1)$ \\
\hline Psicólogo(a) & $126(12)$ \\
\hline Odontólogo & 31 (2.9) \\
\hline Agente comunitário de saúde & $29(2.8)$ \\
\hline Fisioterapeuta & $25(2.4)$ \\
\hline
\end{tabular}




\begin{tabular}{lc}
\hline Variáveis & $\mathrm{n}=1054^{*}$ \\
\hline Farmacêutico(a) & $23(2.2)$ \\
Assistente social & $21(2.0)$ \\
Educador físico & $21(2.0)$ \\
Técnico em radiologia & $18(1.7)$ \\
Nutricionista & $14(1.3)$ \\
Fonoaudiólogo(a) & $10(0.9)$ \\
Terapeuta ocupacional & $8(0.8)$ \\
Biomédico(a) & $7(0,7)$ \\
Anos de profissão - média \pm DP & $15(10.6)$ \\
Horas de trabalho/semana - média \pm DP & $37.6(18.2)$ \\
\hline
\end{tabular}

* amostra total.

Tabela 2. Variáveis sociodemográficas por categoria profissional.

\begin{tabular}{|c|c|c|c|c|c|c|}
\hline & $\begin{array}{l}\text { Médico } \\
(n=364)\end{array}$ & $\begin{array}{l}\text { Enfermeiro } \\
\qquad(n=150)\end{array}$ & $\begin{array}{l}\text { Técnico de } \\
\text { Enfermagem } \\
(n=201)\end{array}$ & $\begin{array}{l}\text { Psicólogo } \\
(n=126)\end{array}$ & $\begin{array}{l}\text { Outros } \\
(n=213)\end{array}$ & $\mathbf{P}$ \\
\hline Sexo-n(\%) & & & & & & $<0,001$ \\
\hline Feminino & 229 (62.9) & $140(93.3)^{*}$ & $188(93.5)^{*}$ & $113(89.7)^{*}$ & $184(86.4)^{*}$ & \\
\hline Masculino & $135(37.1)^{*}$ & $10(6.7)$ & $13(6.5)$ & $13(10.3)$ & $29(13.6)$ & \\
\hline Idade - média $\pm D P$ & $42.2 \pm 11.8^{\mathrm{a}, \mathrm{b}}$ & $42.0 \pm 10.1^{\mathrm{a}, \mathrm{b}}$ & $42.9 \pm 9.4^{b}$ & $40.9 \pm 11.5^{\mathrm{a}, \mathrm{b}}$ & $39.7 \pm 10.5^{\mathrm{a}}$ & 0,027 \\
\hline Cor - n(\%) & & & & & & $<0,001$ \\
\hline Branco & $304(83.5)^{*}$ & $112(74.7)$ & $113(56.2)$ & $109(86.5)^{*}$ & $150(70.4)$ & \\
\hline Pardo & $43(11.8)$ & $28(18.7)$ & $58(28.9)^{*}$ & $11(8.7)$ & $42(19.7)$ & \\
\hline Preto & $8(2.2)$ & $7(4.7)$ & $23(11.4)^{*}$ & $3(2.4)$ & $16(7.5)$ & \\
\hline Amarelo & $5(1.4)$ & $3(2.0)$ & $4(2.0)$ & $2(1.6)$ & $2(0.9)$ & \\
\hline Indígena & $1(0.3)$ & $0(0.0)$ & $1(0.5)$ & $1(0.8)$ & $1(0.5)$ & \\
\hline Outro & $3(0.8)$ & $0(0.0)$ & $2(1.0)$ & $0(0.0)$ & $2(0.9)$ & \\
\hline Estado Civil - n(\%) & & & & & & $<0,001$ \\
\hline $\begin{array}{l}\text { Casado(a)/união } \\
\text { estável }\end{array}$ & $251(69.0)^{*}$ & $75(50)$ & $104(51.7)$ & $67(53.2)$ & $106(49.8)^{* *}$ & \\
\hline $\begin{array}{l}\text { Separado(a)/ } \\
\text { Divorciado(a) }\end{array}$ & $24(6.6)^{* *}$ & $32(21.3)^{*}$ & 34 (16.9)* & $16(12.7)$ & $23(10.8)$ & \\
\hline $\begin{array}{l}\text { Solteiro(a) com } \\
\text { namorado(a) }\end{array}$ & $42(11.5)^{* *}$ & $19(12.7)$ & $33(16.4)$ & $22(17.5)$ & $43(20.2)^{*}$ & \\
\hline $\begin{array}{l}\text { Solteiro(a) sem } \\
\text { namorado(a) }\end{array}$ & $43(11.8)$ & $20(13.3)$ & $27(13.4)$ & $20(15.9)$ & $39(18.3)^{*}$ & \\
\hline \multicolumn{7}{|l|}{ Viúvo } \\
\hline Renda-n(\%) & & & & & & $<0,001$ \\
\hline Até 1.500 reais & $1(0.3)$ & $4(2.7)$ & 24 (11.9)* & $4(3.2)$ & $24(11.3)^{*}$ & \\
\hline $\begin{array}{l}\text { De } 1.500 \text { a } 3.000 \\
\text { reais }\end{array}$ & $3(0.8)$ & $19(12.7)$ & $94(46.8)^{*}$ & $12(9.5)$ & $45(21.1)^{*}$ & \\
\hline
\end{tabular}




\begin{tabular}{|c|c|c|c|c|c|c|}
\hline & $\begin{array}{l}\text { Médico } \\
(n=364)\end{array}$ & $\begin{array}{l}\text { Enfermeiro } \\
\qquad(n=150)\end{array}$ & $\begin{array}{l}\text { Técnico de } \\
\text { Enfermagem } \\
(n=201)\end{array}$ & $\begin{array}{l}\text { Psicólogo } \\
(n=126)\end{array}$ & $\begin{array}{l}\text { Outros } \\
(n=213)\end{array}$ & $\mathbf{P}$ \\
\hline $\begin{array}{l}\text { De } 3.000 \text { a } 5.000 \\
\text { reais }\end{array}$ & $11(3)$ & $50(33.3)^{*}$ & 54 (26.9)* & $24(19.0)$ & $47(22.1)$ & \\
\hline $\begin{array}{l}\text { De } 5.000 \text { a } 10.000 \\
\text { reais }\end{array}$ & $33(9.1)$ & $58(38.7)^{*}$ & $26(12.9)$ & $42(33.3)^{*}$ & $62(29.1)^{*}$ & \\
\hline $\begin{array}{l}\text { Acima de } 10.000 \\
\text { reais }\end{array}$ & $316(86.8)^{*}$ & $19(12.7)$ & $3(1.5)$ & 44 (34.9) & 35 (16.4) & \\
\hline Região - n(\%) & & & & & & $<0,001$ \\
\hline Norte & $6(1,6)$ & $8(5,3) *$ & $3(1,5)$ & $1(0,8)$ & $7(3,3)$ & \\
\hline Nordeste & $51(14)^{*}$ & $17(11,3)$ & $18(9)$ & $8(6,3)$ & $15(7,0)$ & \\
\hline Centro oeste & $5(1,4)^{* *}$ & $3(2,0)$ & $14(7)^{*}$ & $10(7,9)^{*}$ & $12(5,6)$ & \\
\hline Sudeste & $36(9,9) * *$ & $43(28,7)^{*}$ & $87(43,3)^{*}$ & $12(9,5)^{* *}$ & $46(21,6)$ & \\
\hline Sul & $266(73,1)^{*}$ & $79(52,7)^{* *}$ & $79(39,3)^{* *}$ & $95(75,4)^{*}$ & $133(62,4)$ & \\
\hline $\begin{array}{l}\text { Carga horária/ } \\
\text { semana - média } \\
\pm D P\end{array}$ & $42.3 \pm 17.9^{b}$ & $41.4 \pm 16.3^{b}$ & $37.9 \pm 18.9^{b}$ & $28.9 \pm 15.8^{\mathrm{a}}$ & $31.7 \pm 17.1^{\mathrm{a}}$ & $<0.001$ \\
\hline $\begin{array}{l}\text { Anos de profissão - } \\
\text { média } \pm \text { DP }\end{array}$ & $16.2 \pm 11.7^{b}$ & $15.8 \pm 10.3^{\mathrm{a}, \mathrm{b}}$ & $14.6 \pm 8.8^{\mathrm{a}, \mathrm{b}}$ & $14.3 \pm 11.3^{\mathrm{a}, \mathrm{b}}$ & $12.9 \pm 9.3^{\mathrm{a}}$ & 0,005 \\
\hline
\end{tabular}

a,b,c Letras iguais não diferem pelo teste de Tukey a 5\% de significância.

* associação positiva estatisticamente significativa pelo teste dos resíduos ajustados a 5\% de significância.

** associação negativa estatisticamente significativa pelo teste dos resíduos ajustados a 5\% de significância.

Tabela 3. Variáveis relacionadas à exposição ao COVID-19 por categorias profissionais.

\begin{tabular}{|c|c|c|c|c|c|c|c|}
\hline & $\begin{array}{c}\text { Total } \\
(n=1054)\end{array}$ & $\begin{array}{l}\text { Médico } \\
(n=364)\end{array}$ & $\begin{array}{l}\text { Enfermeiro } \\
\qquad(n=150)\end{array}$ & $\begin{array}{l}\text { Técnico de } \\
\text { Enfermagem } \\
(n=201)\end{array}$ & $\begin{array}{l}\text { Psicólogo } \\
(n=126)\end{array}$ & $\begin{array}{l}\text { Outros } \\
(n=213)\end{array}$ & $p$ \\
\hline $\begin{array}{l}\text { Diagnóstico COVID } \\
\text { confirmado-n (\%) }\end{array}$ & & & & & & & 0,001 \\
\hline Sim & $61(5,8)$ & $23(6,3)$ & $12(8,0)$ & $20(10,0)^{*}$ & $2(1,6)$ & $4(1,9)$ & \\
\hline Não & $993(94,2)$ & $341(93,7)$ & $138(92,0)$ & $181(90,0)$ & $124(98,4)^{*}$ & $209(98,1)^{*}$ & \\
\hline $\begin{array}{l}\text { Grupo de risco para } \\
\text { COVID - n (\%) }\end{array}$ & & & & & & & 0,002 \\
\hline Sim & $274(26)$ & $77(21,2)$ & $43(28,7)$ & $73(36,3)^{*}$ & $27(21,4)$ & $54(25,4)$ & \\
\hline Não & $780(74)$ & $287(78,8)^{*}$ & $107(71,3)$ & $128(63,7)$ & $99(78,6)$ & $159(74,6)$ & \\
\hline $\begin{array}{l}\text { Assistência a } \\
\text { pacientes COVID } \\
-\mathrm{n}(\%)\end{array}$ & & & & & & & $<0,001$ \\
\hline $\begin{array}{l}\text { Sim, no } \\
\text { atendimento direto }\end{array}$ & $455(43,2)$ & $176(48,4)^{*}$ & $88(58,7)^{*}$ & $119(59,2)^{*}$ & $17(13,5)^{* *}$ & $55(25,8)^{* *}$ & \\
\hline $\begin{array}{l}\text { Sim, no } \\
\text { atendimento } \\
\text { indireto }\end{array}$ & $261(24,8)$ & $83(22,8)$ & $43(28,7)$ & $45(22,4)$ & $30(23,8)$ & $60(28,2)$ & \\
\hline Não & $338(32,1)$ & $105(28,8)$ & $105(28,8)$ & $19(12,7)^{* *}$ & $37(18,4)^{* *}$ & $79(62,7)^{*}$ & \\
\hline
\end{tabular}




\begin{tabular}{|c|c|c|c|c|c|c|c|}
\hline & $\begin{array}{c}\text { Total } \\
(n=1054)\end{array}$ & $\begin{array}{l}\text { Médico } \\
(n=364)\end{array}$ & $\begin{array}{l}\text { Enfermeiro } \\
\qquad(n=150)\end{array}$ & $\begin{array}{l}\text { Técnico de } \\
\text { Enfermagem } \\
(n=201)\end{array}$ & $\begin{array}{l}\text { Psicólogo } \\
(n=126)\end{array}$ & $\begin{array}{l}\text { Outros } \\
(n=213)\end{array}$ & p \\
\hline $\begin{array}{l}\text { Coação } \\
\text { atividade sem } \\
\text { capacitação-n(\%)*** }\end{array}$ & & & & & & & 0,158 \\
\hline Sim & $103(14,3)$ & $34(13,1)$ & $16(12,2)$ & $33(20,1)^{*}$ & $4(8,5)$ & $16(13,8)$ & \\
\hline Não & $615(85,7)$ & $226(86,9)$ & $115(87,8)$ & $131(79,9)^{* *}$ & $43(91,5)$ & $100(86,2)$ & \\
\hline $\begin{array}{l}\text { Coação atividade } \\
\text { indesejada }-n(\%)^{* * *}\end{array}$ & & & & & & & 0,800 \\
\hline Sim & $131(18,2)$ & $46(17,7)$ & $22(16,8)$ & $35(21,3)$ & $7(14,9)$ & $21(18,1)$ & \\
\hline Não & $587(81,8)$ & $214(82,3)$ & $109(83,2)$ & $129(78,7)$ & $40(85,1)$ & $95(81,9)$ & \\
\hline $\begin{array}{l}\text { Disponibilização EPI } \\
-\mathrm{n}(\%)^{* * *}\end{array}$ & & & & & & & 0,001 \\
\hline $\begin{array}{l}\text { Sempre/Maioria } \\
\text { das vezes }\end{array}$ & $609(81,1)$ & $230(86,8) *$ & $107(80,5)$ & $137(77)$ & $41(78,8)$ & $94(76,4)$ & \\
\hline Às vezes & $99(13,2)$ & $27(10,2)$ & $20(15)$ & $31(17,4)$ & $2(3,8)^{* *}$ & $19(15,4)$ & \\
\hline $\begin{array}{l}\text { Quase nunca/ } \\
\text { Nunca }\end{array}$ & $43(5,7)$ & $8(3)^{* *}$ & $6(4,5)$ & $10(5,6)$ & $9(17,3)^{*}$ & $10(8,1)$ & \\
\hline $\begin{array}{l}\text { Afastamento } \\
\text { proteção familiares } \\
-\mathrm{n}(\%)\end{array}$ & & & & & & & 0,682 \\
\hline Sim & $252(35,1)$ & $99(38,1)$ & $41(31,3)$ & $55(33,5)$ & $15(31,9)$ & $42(36,2)$ & \\
\hline Não & $466(64,9)$ & $161(61,9)$ & $90(68,7)$ & $109(66,5)$ & $32(68,1)$ & $74(63,8)$ & \\
\hline
\end{tabular}

* associação positiva estatisticamente significativa pelo teste dos resíduos ajustados a $5 \%$ de significância.

** associação negativa estatisticamente significativa pelo teste dos resíduos ajustados a $5 \%$ de significância.

*** nos casos onde não há a amostra total é porque essa questão não se aplicava a todos os participantes.

Tabela 4. Perfil clínico por categorias profissionais.

\begin{tabular}{|c|c|c|c|c|c|c|c|}
\hline & $\begin{array}{c}\text { Total } \\
(n=1054)\end{array}$ & $\begin{array}{l}\text { Médico } \\
(n=364)\end{array}$ & $\begin{array}{l}\text { Enfermeiro } \\
\qquad(n=150)\end{array}$ & $\begin{array}{l}\text { Técnico de } \\
\text { Enfermagem } \\
(n=201)\end{array}$ & $\begin{array}{l}\text { Psicólogo } \\
(n=126)\end{array}$ & $\begin{array}{l}\text { Outros } \\
(n=213)\end{array}$ & $p$ \\
\hline $\begin{array}{l}\text { Tratamento atual } \\
\text { saúde mental - } \\
\mathrm{n}(\%)\end{array}$ & & & & & & & 0,001 \\
\hline Sim & $259(24,6)$ & $107(29,4)^{*}$ & $26(17,3)$ & $43(21,4)$ & $39(31,0)$ & $44(20,7)$ & \\
\hline $\begin{array}{l}\text { Não e não sinto } \\
\text { que preciso }\end{array}$ & $565(53,6)$ & $204(56)$ & $81(54)$ & $102(50,7)$ & $65(51,6)$ & $113(53,1)$ & \\
\hline $\begin{array}{l}\text { Não, mas sinto } \\
\text { que preciso }\end{array}$ & $230(21,8)$ & $53(14,6)$ & $43(28,7)^{*}$ & $56(27,9)^{*}$ & $22(17,5)$ & $56(26,3)$ & \\
\hline $\begin{array}{l}\text { História de } \\
\text { diagnóstico } \\
\text { psiquiátrico - n(\%) }\end{array}$ & & & & & & & 0,031 \\
\hline Sim & $357(33,9)$ & $146(40,1)^{*}$ & $44(29,3)$ & $65(32,3)$ & $41(32,5)$ & $61(28,6)$ & \\
\hline Não & $697(66,1)$ & $218(59,9)$ & $106(70,7)$ & $136(67,7)$ & $85(67,5)$ & $152(71,4)$ & \\
\hline
\end{tabular}




\begin{tabular}{|c|c|c|c|c|c|c|c|}
\hline & $\begin{array}{c}\text { Total } \\
(n=1054)\end{array}$ & $\begin{array}{l}\text { Médico } \\
(n=364)\end{array}$ & $\begin{array}{l}\text { Enfermeiro } \\
\qquad(n=150)\end{array}$ & $\begin{array}{c}\text { Técnico de } \\
\text { Enfermagem } \\
(n=201)\end{array}$ & $\begin{array}{l}\text { Psicólogo } \\
(n=126)\end{array}$ & $\begin{array}{l}\text { Outros } \\
(n=213)\end{array}$ & p \\
\hline $\begin{array}{l}\text { História de } \\
\text { ideação suicida - } \\
\mathrm{n}(\%)^{* *}\end{array}$ & & & & & & & 0,253 \\
\hline Sim & $246(23,3)$ & $77(22,8)$ & $43(31,9)$ & $51(28,2)$ & $26(22,6)$ & $49(26,1)$ & \\
\hline Não & $711(67,5)$ & $261(77,2)$ & $92(68,1)$ & $130(71,8)$ & $89(77,4)$ & $139(73,9)$ & \\
\hline $\begin{array}{l}\text { Ideação suicida no } \\
\text { último mês - } \\
\mathrm{n}(\%)^{* *}\end{array}$ & & & & & & & 0,099 \\
\hline Sim & $79(7,5)$ & $24(7,1)$ & $14(10,4)$ & $15(8,3)$ & $4(3,5)$ & $22(11,7)$ & \\
\hline Não & $878(83,3)$ & $314(92,9)$ & $121(89,6)$ & $166(91,7)$ & $111(96,5)$ & $166(88,3)$ & \\
\hline $\begin{array}{l}\text { História de } \\
\text { tentativa de } \\
\text { suicídio** - n (\%) }\end{array}$ & & & & & & & $<0,001$ \\
\hline Sim & $60(5,7)$ & $8(2,4)$ & $13(9,6)$ & $23(12,7)^{*}$ & $4(3,5)$ & $12(6,4)$ & \\
\hline Não & $897(85,1)$ & $330(97,6)^{*}$ & $122(90,4)$ & $158(87,3)$ & $111(96,5)$ & $176(93,6)$ & \\
\hline $\begin{array}{l}\text { Considera-se em } \\
\text { Burnout - n (\%) }\end{array}$ & & & & & & & $<0,001$ \\
\hline Sim & $323(30,6)$ & $94(25,8)$ & $48(32,0)$ & $81(40,3)^{*}$ & $17(13,5)$ & $83(39) *$ & \\
\hline Não & $731(69,4)$ & $270(74,2)^{*}$ & $102(68,0)$ & $120(59,7)$ & $109(86,5)^{*}$ & $130(61)$ & \\
\hline $\begin{array}{l}\text { Trauma na } \\
\text { infância - n (\%)** }\end{array}$ & & & & & & & $<0,001$ \\
\hline Sim & $283(26,9)$ & $66(19,5)$ & $42(31,1)$ & $74(40,9)^{*}$ & $43(37,4)^{*}$ & $58(30,9)$ & \\
\hline Não & $674(63,9)$ & $272(80,5)^{*}$ & $93(68,9)$ & $107(59,1)$ & $72(62,6)$ & $130(69,1)$ & \\
\hline
\end{tabular}

* associação positiva estatisticamente significativa pelo teste dos resíduos ajustados a $5 \%$ de significância.

** nos casos onde não há a amostra total é porque alguns participantes não informaram sobre a variável em questão.

Tabela 5. Escores dos instrumentos.

\begin{tabular}{lcccccc}
\hline Variáveis & $\begin{array}{c}\text { Médico } \\
(n=346)\end{array}$ & $\begin{array}{c}\text { Enfermeiro } \\
(n=150)\end{array}$ & $\begin{array}{c}\text { Técnico de } \\
\text { Enfermagem } \\
(n=201)\end{array}$ & $\begin{array}{c}\text { Psicólogo } \\
(n=126)\end{array}$ & $\begin{array}{c}\text { Outros } \\
(n=213)\end{array}$ & $\begin{array}{c}\text { TOTAL } \\
(n=1054)\end{array}$ \\
\hline PB & $48,2 \pm 19,9^{\mathrm{a}, \mathrm{b}}$ & $53,7 \pm 20,1^{\mathrm{b}, \mathrm{c}}$ & $58,4 \pm 20,9^{\mathrm{c}}$ & $44,2 \pm 17,4^{\mathrm{a}}$ & $52,8 \pm 19,6^{\mathrm{b}, \mathrm{c}}$ & $51,4 \pm 20,2$ \\
PB $\geq 50$ & $166(45,6)^{* *}$ & $90(60,0)$ & $137(68,2)^{*}$ & $50(39,7)^{* *}$ & $120(56,3)$ & $563(53,4)$ \\
WB & $44,2 \pm 19,1^{\mathrm{a}, \mathrm{b}}$ & $48,5 \pm 20,2^{\mathrm{b}, \mathrm{c}}$ & $51,0 \pm 21,1^{\mathrm{c}}$ & $41,2 \pm 16,7^{\mathrm{a}}$ & $47,1 \pm 20,2^{\mathrm{b}, \mathrm{c}}$ & $46,3 \pm 19,8$ \\
WB $\geq 50$ & $156(42,9)^{* *}$ & $78(52,0)$ & $117(58,2)^{*}$ & $48(38,1)^{* *}$ & $102(47,9)$ & $501(47,5)$ \\
CB & $37,0 \pm 23,6^{\mathrm{b}}$ & $40,0 \pm 23,2^{\mathrm{b}}$ & $39,3 \pm 26,6^{\mathrm{b}}$ & $29,3 \pm 19,3^{\mathrm{a}}$ & $40,0 \pm 22,6^{\mathrm{b}}$ & $37,6 \pm 23,7$ \\
$\mathrm{CB} \geq 50$ & $114(31,3)$ & $54(36,0)$ & $71(35,3)$ & $17(13,5)^{* *}$ & $77(36,2)$ & $333(31,6)$ \\
PHQ & $8,5 \pm 6,1^{\mathrm{a}}$ & $10,8 \pm 6,8^{\mathrm{b}}$ & $13,0 \pm 7,3^{\mathrm{c}}$ & $7,4 \pm 5,4^{\mathrm{a}}$ & $11,2 \pm 6,8^{\mathrm{b}, \mathrm{c}}$ & $10,1 \pm 6,8$ \\
PHQ $\geq 9$ & $150(42,9)^{* *}$ & $81(55,9)$ & $134(68,7)^{*}$ & $41(33,6)^{* *}$ & $123(60,6)^{*}$ & $529(50,2)$ \\
\hline
\end{tabular}

a,b,c Letras iguais não diferem pelo teste de Tukey a 5\% de significância.

* associação positiva estatisticamente significativa pelo teste dos resíduos ajustados a 5\% de significância.

** associação negativa estatisticamente significativa pelo teste dos resíduos ajustados a $5 \%$ de significância. 
Tabela 6. Escores de burnout e PHQ da amostra total de acordo com o nível de exposição ao COVID-19.

\begin{tabular}{|c|c|c|c|c|}
\hline Variáveis & $\begin{array}{c}\text { Assistência direta } \\
(n=455)\end{array}$ & $\begin{array}{l}\text { Assistência indireta } \\
\qquad(n=261)\end{array}$ & $\begin{array}{l}\text { Não prestaram assistência } \\
\qquad(\mathrm{n}=338)\end{array}$ & $p$ \\
\hline PB & $55,4 \pm 20,5^{b}$ & $49,9 \pm 19,0^{a}$ & $47,1 \pm 19,9^{a}$ & $<0,001$ \\
\hline$P B \geq 50$ & $61,3(279)^{*}$ & $50,2(131)$ & $45,3(153)^{* *}$ & $<0,001$ \\
\hline WB & $50,5 \pm 19,9^{b}$ & $44,9 \pm 18,4^{a}$ & $41,9 \pm 19,6^{a}$ & $<0,001$ \\
\hline$W B \geq 50$ & $57,6(262)^{*}$ & 44,1 (115) & $36,7(124)^{* *}$ & $<0,001$ \\
\hline $\mathrm{CB}$ & $39,6 \pm 24,2^{b}$ & $37,0 \pm 21,7^{a, b}$ & $35,1 \pm 24,2^{a}$ & 0,028 \\
\hline$C B \geq 50$ & $35,8(163)^{*}$ & $29,9(78)$ & $27,2(92)^{* *}$ & 0,029 \\
\hline PHQ & $10,9 \pm 6,8^{b}$ & $9,9 \pm 6,4^{a, b}$ & $9,0 \pm 6,8^{a}$ & $<0,001$ \\
\hline $\mathrm{PHQ} \geq 9$ & $58(257)^{*}$ & $52,4(131)$ & $43,8(141)^{* *}$ & $<0,001$ \\
\hline
\end{tabular}

a,b Letras iguais não diferem pelo teste de Tukey a 5\% de significância.

* associação positiva estatisticamente significativa pelo teste dos resíduos ajustados a $5 \%$ de significância.

** associação negativa estatisticamente significativa pelo teste dos resíduos ajustados a 5\% de significância.

\section{Discussão}

Os principais achados desta investigação demonstram índices preocupantes de sintomas depressivos e indicativos de burnout em PS, sobretudo em técnicos de enfermagem. Os resultados de nossa pesquisa vão ao encontro de estudos que mostram a vulnerabilidade dos PS ao sofrimento emocional ${ }^{19,20}$. Este é um público habitualmente acometido por sintomas de depressão e ansiedade. O contexto da pandemia pode ser um fator estressor que alavanque a prevalência destes transtornos ${ }^{4}$. De fato, metade da amostra total apresentou sintomas sugestivos de alto nível de burnout e de quadro depressivo clinicamente significativo, sendo os índices mais preocupantes encontrados entre os técnicos de enfermagem. Estes profissionais, juntamente com os enfermeiros, foram os que mais referiram não estarem em tratamento para saúde mental, apesar de reconhecerem que se beneficiariam de acompanhamento, o que torna a situação destas categorias profissionais ainda mais crítica.

Em nossa amostra, embora as taxas de assistência direta a pacientes com suspeita ou diagnóstico confirmado de Covid-19 tenham sido semelhantes entre médicos, enfermeiros e técnicos de enfermagem, a categoria dos técnicos foi a que apresentou a maior proporção de profissionais contaminados e indivíduos do grupo de alto risco para Covid-19. Um dos aspectos que pode explicar este resultado é a caracterização distinta das atividades de cada área profissional, sendo possível que a presença mais constante dos técnicos junto aos pacientes na assistência direta os faça ficar mais expostos e mais desgastados emocionalmente. Ademais, a pesquisa foi realizada no início da pandemia, quando alguns protocolos de proteção e manejo dos casos ainda estavam sendo estabelecidos e aprendidos, o que provavelmente tenha sido um fator adicional de estresse.

Vale destacar que a carga horária semanal de trabalho também não foi diferente entre médicos, enfermeiros e técnicos de enfermagem, sendo inferior entre os psicólogos e no grupo dos outros profissionais da saúde. Portanto, o que destacamos diz respeito não somente à carga de trabalho, mas à maneira como ela é distribuída entre as diferentes profissões. Além disso, a maior proximidade dos técnicos de enfermagem 
com os pacientes pode deixá-los mais mobilizados com questões emocionais e físicas que emergem desse contato profissional. Outro fator que pode estar relacionado à maior ocorrência de burnout e depressão nos técnicos de enfermagem é a maior prevalência de traumas na infância, de modo que os estressores atuais podem ser gatilhos em um psiquismo já vulnerável por exposições anteriores. Ainda há que se considerar a possível associação de burnout com vulnerabilidades socioeconômicas, sendo os técnicos de enfermagem um grupo com menor renda em relação aos demais profissionais. Estes dados sugerem a importância da atenção a condições potencialmente predeterminantes que, mesmo que mais precocemente instaladas na trajetória destes indivíduos, parecem ter deixado estes profissionais mais vulneráveis ao burnout no cenário pandêmico.

Nossos achados vão ao encontro daqueles apontados por estudos prévios que encontraram taxas elevadas de burnout e depressão entre PS no contexto da pandemia, sobretudo naqueles envolvidos na assistência direta aos indivíduos contaminados (linha de frente) e na área da enfermagem. Uma revisão sistemática realizada em 2020 analisou um total de 13 estudos e encontrou que a saúde mental dos PS que atuam na linha de frente fica comprometida em tempos de pandemia por apresentar níveis moderados a altos de ansiedade, depressão, nervosismo e insônia e, em menor grau, estresse ${ }^{25}$. Outra revisão sistemática, publicada já em 2021, mostrou que estudos quantitativos europeus e americanos relataram níveis moderados a altos de estresse, ansiedade, depressão, distúrbios do sono e burnout, com diversas estratégias de enfrentamento e sintomas mais frequentes e intensos entre mulheres e enfermeiras. De acordo com essa revisão, o impacto psicológico foi maior nos PS da linha de frente e superior ao relatado na região asiática ${ }^{45}$.

Nossa amostra teve 5,7\% dos indivíduos com história de tentativa de suicídio prévia e 23,3\% com histórico de ideação suicida na vida. Sabe-se que tentativas de suicídio anteriores e transtornos mentais são fatores de risco conhecidos para suicídio ${ }^{46}$. Além disso, 7,5\% dos indivíduos na nossa amostra relataram ideação suicida no último mês. Estudos têm mostrado que a pandemia aumentou a prevalência de ideação suicida. Um estudo feito na população geral nos Estados Unidos em 2020 mostrou que a porcentagem de entrevistados que relataram ter considerado seriamente o suicídio 30 dias antes de completar a pesquisa $(10,7 \%)$ foi significativamente maior entre os entrevistados com idade entre 18-24 anos (25,5\%), grupos raciais / étnicos minoritários (18,6\% em entrevistados hispânicos e 15,1\% em entrevistados não hispânicos negros), cuidadores não remunerados de adultos autorreferidos $(30,7 \%)$ e trabalhadores essenciais $(21,7 \%)^{47}$.

Entre os pontos fortes deste estudo, podemos citar o tamanho de amostra, que permite corrigir nossos achados para vários fatores de confusão. Além disso, de acordo com nosso conhecimento até o momento, trata-se de um dos primeiros estudos a avaliar o impacto da pandemia na saúde mental dos profissionais da saúde do Brasil. Outrossim, propusemo-nos a incluir a diversidade multiprofissional dos atores da área da saúde.

No entanto, embora tenhamos coletado muitas categorias de profissionais da saúde, algumas delas tiveram menor distribuição amostral, tendo havido a necessidade de criar o grupo denominado "outros profissionais da saúde". Além disso, o fato de ter sido online, com amostra por conveniência e recrutamento via snowball, pode ter atraído voluntários já bastante engajados, interessados no assunto e com acesso à internet. Nesse sentido, existe o risco de que os indivíduos mais vulneráveis tenham sido excluídos ${ }^{48}$. 
Considerando o perfil dos indivíduos que foram excluídos por não completarem as questões relacionadas à avaliação do nível de burnout, é possível que obtivéssemos escores mais elevados tanto para burnout, quanto para depressão. De fato, esses indivíduos foram mais jovens, com menos tempo de profissão, menor renda, com maior proporção pertencente à grupo de alto risco para Covid-19 e às categorias profissionais de técnico de enfermagem, biomédico e biotecnólogo.

Cabe ainda mencionar que nosso estudo foi realizado no início da pandemia, e é possível que encontrássemos escores de burnout e depressão mais elevados após um tempo mais prolongado de pandemia, especialmente entre os profissionais da linha de frente e entre os técnicos de enfermagem.

\section{Conclusão}

Em suma, os achados do presente estudo demarcam a vulnerabilidade dos PS ao sofrimento emocional no contexto de atendimento à Covid-19. A amostra deste estudo, composta por várias categorias profissionais, evidenciou sintomas sugestivos de alto nível de burnout e de quadro depressivo clinicamente significativo, sendo estes índices mais alarmantes dentre os técnicos de enfermagem. Intervenções direcionadas especificamente a esta população são urgentes.

Nossos dados sugerem que esses profissionais precisam ter a sua saúde mental monitorada regularmente, sobretudo no que se refere aos escores de depressão, ansiedade, esgotamento e risco de suicídio. Tratamentos psiquiátricos devem ser fornecidos para aqueles que apresentarem problemas de saúde mental mais graves. Especificamente no que refere ao cuidado com a saúde mental dos profissionais da saúde no contexto da Covid-19, torna-se importante identificar fatores psicossociais que conferem uma condição de maior vulnerabilidade, como traumas prévios e dificuldades socioeconômicas.

\section{Referências}

1. Adams JG, Walls RM. Supporting the Health Care Workforce During the COVID-19 Global Epidemic. JAMA. 2020;323(15):1439-40.

2. Zhonghua Liu Xing Bing Xue Za Zhi. [The epidemiological characteristics of an outbreak of 2019 novel coronavirus diseases (COVID-19) in China]. 2020;41(2):145-51.

3. Huang JZ, Han MF, Luo TD, Ren AK, Zhou XP. [Mental health survey of 230 medical staff in a tertiary infectious disease hospital for COVID-19]. Zhonghua Lao Dong Wei Sheng Zhi Ye Bing Za Zhi. 2020;38(0):E001.

4. Ornell F, Halpern SC, Kessler FHP, Narvaez JCM. The Impact of the COVID-19 Pandemic on the Mental Health of Healthcare Professionals. Cadernos de saude publica. 2020;36(4).

5. Ornell F, Schuch JB, Sordi AO, Kessler FHP. "Pandemic Fear" and COVID-19: Mental Health Burden and Strategies. Revista brasileira de psiquiatria (Sao Paulo, Brazil : 1999). 2020;42(3).

6. Bao Y, Sun Y, Meng S, Shi J, Lu L. 2019-nCoV epidemic: address mental health care to empower society. Lancet. 2020;395(10224):e37-e8.

7. Shigemura J, Ursano RJ, Morganstein JC, Kurosawa M, Benedek DM. Public responses to the novel 2019 coronavirus (2019-nCoV) in Japan: Mental health consequences and target populations. Psychiatry Clin Neurosci. 2020;74(4):281-2. 
8. Kang L, Li Y, Hu S, Chen M, Yang C, Yang BX, et al. The mental health of medical workers in Wuhan, China dealing with the 2019 novel coronavirus. Lancet Psychiatry. 2020;7(3):e14.

9. Xiang YT, Yang Y, Li W, Zhang L, Zhang Q, Cheung T, et al. Timely mental health care for the 2019 novel coronavirus outbreak is urgently needed. Lancet Psychiatry. 2020;7(3):228-9.

10. Malta M, Rimoin AW, Strathdee SA. The coronavirus 2019-nCoV epidemic: Is hindsight 20/20? EClinicalMedicine. 202020.

11. Mihailescu M, Neiterman E. A scoping review of the literature on the current mental health status of physicians and physicians-in-training in North America. BMC Public Health. 2019;19(1):1363.

12. Lai J, Ma S, Wang Y, Cai Z, Hu J, Wei N, et al. Factors Associated With Mental Health Outcomes Among Health Care Workers Exposed to Coronavirus Disease 2019. JAMA Netw Open. 2020;3(3).

13. Bansal P, Bingemann TA, Greenhawt M, Mosnaim G, Nanda A, Oppenheimer J, et al. Clinician Wellness During the COVID-19 Pandemic: Extraordinary Times and Unusual Challenges for the Allergist/Immunologist. J Allergy Clin Immunol Pract. 2020;8(6):1781-90.e3.

14. Wang C, Pan R, Wan X, Tan Y, Xu L, Ho CS, et al. Immediate Psychological Responses and Associated Factors during the Initial Stage of the 2019 Coronavirus Disease (COVID-19) Epidemic among the General Population in China. Int J Environ Res Public Health. 2020;17(5).

15. Shaw SC. Hopelessness, helplessness and resilience: The importance of safeguarding our trainees' mental wellbeing during the COVID-19 pandemic. Nurse Educ Pract. 2020;44:102780.

16. Xiang Y-T, Yang Y, Li W, Zhang L, Zhang Q, Cheung T, et al. Timely mental health care for the 2019 novel coronavirus outbreak is urgently needed. The Lancet Psychiatry. 2020;7(3):228-9.

17. Li Z, Ge J, Yang M, Feng J, Qiao M, Jiang R, et al. Vicarious traumatization in the general public, members, and non-members of medical teams aiding in COVID-19 control. Brain Behav Immun. 2020.

18. Lee AM, Wong JG, McAlonan GM, Cheung V, Cheung C, Sham PC, et al. Stress and psychological distress among SARS survivors 1 year after the outbreak. Can J Psychiatry. 2007;52(4):233-40.

19. Lee SM, Kang WS, Cho AR, Kim T, Park JK. Psychological impact of the 2015 MERS outbreak on hospital workers and quarantined hemodialysis patients. Compr Psychiatry. 2018;87:123-7.

20. Kim JS, Choi JS. Factors Influencing Emergency Nurses' Burnout During an Outbreak of Middle East Respiratory Syndrome Coronavirus in Korea. Asian Nurs Res (Korean Soc Nurs Sci). 2016;10(4):295-9.

21. Cai H, Tu B, Ma J, Chen L, Fu L, Jiang Y, et al. Psychological Impact and Coping Strategies of Frontline Medical Staff in Hunan Between January and March 2020 During the Outbreak of Coronavirus Disease 2019 (COVID-19) in Hubei, China. Med Sci Monit. 2020;26:e924171.

22. Zhang WR, Wang K, Yin L, Zhao WF, Xue Q, Peng M, et al. Mental Health and Psychosocial Problems of Medical Health Workers during the COVID-19 Epidemic in China. Psychother Psychosom. 2020;89(4):24250.

23. Bohlken J, Schömig F, Lemke MR, Pumberger M, Riedel-Heller SG. [COVID-19 Pandemic: Stress Experience of Healthcare Workers - A Short Current Review]. Psychiatr Prax. 2020;47(4):190-7.

24. Vizheh M, Qorbani M, Arzaghi SM, Muhidin S, Javanmard Z, Esmaeili M. The mental health of healthcare workers in the COVID-19 pandemic: A systematic review. J Diabetes Metab Disord. 2020:1-12.

25. Pappa S, Ntella V, Giannakas T, Giannakoulis VG, Papoutsi E, Katsaounou P. Prevalence of depression, anxiety, and insomnia among healthcare workers during the COVID-19 pandemic: A systematic review and meta-analysis. Brain Behav Immun. 2020;88:901-7. 
26. Salazar CA, Tomko RL, Akbar SA, Squeglia LM, McClure EA. Medical Cannabis Use among Adults in the Southeastern United States. Cannabis. 2019;2(1):53-65.

27. Luo M, Guo L, Yu M, Jiang W, Wang H. The psychological and mental impact of coronavirus disease 2019 (COVID-19) on medical staff and general public - A systematic review and meta-analysis. Psychiatry Res. 2020;291:113190.

28. Muller AE, Hafstad EV, Himmels JPW, Smedslund G, Flottorp S, Stensland S, et al. The mental health impact of the covid-19 pandemic on healthcare workers, and interventions to help them: A rapid systematic review. Psychiatry Res. 2020;293:113441.

29. Xiao H, Zhang Y, Kong D, Li S, Yang N. The Effects of Social Support on Sleep Quality of Medical Staff Treating Patients with Coronavirus Disease 2019 (COVID-19) in January and February 2020 in China. Med Sci Monit. 2020;26:e923549.

30. Trumello C, Bramanti SM, Ballarotto G, Candelori C, Cerniglia L, Cimino S, et al. Psychological Adjustment of Healthcare Workers in Italy during the COVID-19 Pandemic: Differences in Stress, Anxiety, Depression, Burnout, Secondary Trauma, and Compassion Satisfaction between Frontline and Non-Frontline Professionals. Int J Environ Res Public Health. 2020;17(22).

31. Civantos AM, Bertelli A, Gonçalves A, Getzen E, Chang C, Long $Q$, et al. Mental health among head and neck surgeons in Brazil during the COVID-19 pandemic: A national study. Am J Otolaryngol. 2020;41(6):102694.

32. Campos JADB, Martins BG, Campos LA, de Fátima Valadão-Dias F, Marôco J. Symptoms related to mental disorder in healthcare workers during the COVID-19 pandemic in Brazil. Int Arch Occup Environ Health. 2021.

33. De Boni RB, Balanzá-Martínez V, Mota JC, Cardoso TA, Ballester P, Atienza-Carbonell B, et al. Depression, Anxiety, and Lifestyle Among Essential Workers: A Web Survey From Brazil and Spain During the COVID-19 Pandemic. J Med Internet Res. 2020;22(10):e22835.

34. Marcon G, Massaro Carneiro Monteiro G, Ballester P, Cassidy RM, Zimerman A, Brunoni AR, et al. Who attempts suicide among medical students? Acta Psychiatr Scand. 2020;141(3):254-64.

35. Santos IS, Tavares BF, Munhoz TN, Almeida LS, Silva NT, Tams BD, et al. [Sensitivity and specificity of the Patient Health Questionnaire-9 (PHQ-9) among adults from the general population]. Cad Saude Publica. 2013;29(8):1533-43.

36. Kroenke K, Spitzer RL, Williams JB. The PHQ-9: validity of a brief depression severity measure. J Gen Intern Med. 2001;16(9):606-13.

37. Santos IS, Tavares BF, Munhoz TN, de Almeida LSP, da Silva NTB, Tams BD, et al. Sensitivity and Specificity of the Patient Health Questionnaire-9 (PHQ-9) Among Adults From the General Population. Cadernos de saude publica. $2013 ; 29(8)$.

38. Matias AGC, Fonseca MA, Gomes MLF, Matos MAA. Indicators of depression in elderly and different screening methods. Einstein (São Paulo). 2016;14(1):6-11.

39. Kristensen TS, Borritz M, Villadsen E, Christensen KB. The Copenhagen Burnout Inventory: A new tool for the assessment of burnout. Work \& Stress. 2007;19:192-207.

40. Maslach C, Leiter MP. Understanding the burnout experience: recent research and its implications for psychiatry. World Psychiatry. 152016. p. 103-11.

41. Campos JADBC, M.S. Maroco, J. Copenhagen Burnout Inventory - student version: adaptation and transcultural validation for Portugal and Brazil. Psicologia: Reflexão e Crítica. 2013;26(1):87-97.

42. Posner K, Brown GK, Stanley B, Brent DA, Yershova KV, Oquendo MA, et al. The Columbia-Suicide Severity 
Rating Scale: initial validity and internal consistency findings from three multisite studies with adolescents and adults. Am J Psychiatry. 2011;168(12):1266-77.

43. Andrade SV, Sesso R, Diniz DH. Hopelessness, Suicide Ideation, and Depression in Chronic Kidney Disease Patients on Hemodialysis or Transplant Recipients. Jornal brasileiro de nefrologia : 'orgao oficial de Sociedades Brasileira e Latino-Americana de Nefrologia. 2015;37(1).

44. Andrade SV, Sesso R, Diniz DH. Hopelessness, suicide ideation, and depression in chronic kidney disease patients on hemodialysis or transplant recipients. J Bras Nefrol. 2015;37(1):55-63.

45. Danet Danet A. Psychological impact of COVID-19 pandemic in Western frontline healthcare professionals. A systematic review. Med Clin (Barc). 2021.

46. CDC. Risk and Protective Factors. Centers for Disease Control and Pevention; 2021.

47. Czeisler M, Lane RI, Petrosky E, Wiley JF, Christensen A, Njai R, et al. Mental Health, Substance Use, and Suicidal Ideation During the COVID-19 Pandemic - United States, June 24- 30, 2020. MMWR Morb Mortal Wkly Rep. 2020;69(32):1049-57.

48. Pierce M, McManus S, Jessop C, John A, Hotopf M, Ford T, et al. Says who? The significance of sampling in mental health surveys during COVID-19. Lancet Psychiatry. 2020;7(7):567-8.

Contribuição do autor: Carolina Meira Moser - Análise estatística, Aquisição de financiamento, Coleta de Dados, Conceitualização, Gerenciamento de Recursos, Gerenciamento do Projeto, Investigação, Metodologia, Redação - Preparação do original, Redação - Revisão e Edição, Validação, Visualização;

Gabriela Carneiro Monteiro - Coleta de Dados, Investigação, Redação - Preparação do original, Visualização; Joana Correa de Magalhães Narvaez - Coleta de Dados, Conceitualização, Investigação, Redação - Preparação do original, Visualização;

Felipe Ornell - Coleta de Dados, Investigação, Redação - Preparação do original, Visualização;re - RS - Brasil Vitor Crestani Calegaro - Redação - Revisão e Edição, Supervisão, Visualização;

Ana Margareth Siqueira Bassols - Redação - Preparação do original, Visualização;

Pricilla Braga Laskoski - Investigação, Redação - Preparação do original, Visualização;

Simone Hauck - Análise estatística, Aquisição de financiamento, Coleta de Dados, Conceitualização, Gerenciamento de Recursos, Gerenciamento do Projeto, Investigação, Metodologia, Redação - Preparação do original, Redação - Revisão e Edição, Supervisão, Validação, Visualização.

\section{Correspondência}

\section{Carolina Meira Moser}

cmeiramoser@gmail.com

Submetido em: 20/03/2021

Aceito em: 28/05/2021 